Published in final edited form as:

Complement Ther Clin Pract. 2016 August ; 24: 19-31. doi:10.1016/j.ctcp.2016.04.005.

\title{
Massage therapy research review
}

\author{
Tiffany Fielda,b, \\ aTouch Research institute, University of Miami/Miller School of Medicine, USA

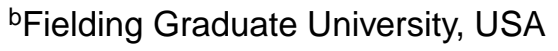

\section{Abstract}

In this review, massage therapy has been shown to have beneficial effects on varying conditions including prenatal depression, preterm infants, full-term infants, autism, skin conditions, pain syndromes including arthritis and fibromyalgia, hypertension, autoimmune conditions including asthma and multiple sclerosis, immune conditions including HIV and breast cancer and aging problems including Parkinson's and dementia. Although many of the studies have involved comparisons between massage therapy and standard treatment control groups, several have compared different forms of massage (e.g. Swedish versus Thai massage), and different active therapies such as massage versus exercise. Typically, the massage therapy groups have experienced more positive effects than the control or comparison groups. This may relate to the massage therapy providing more stimulation of pressure receptors, in turn enhancing vagal activity and reducing Cortisol levels. Some of the researchers have assessed physical, physiological and biochemical effects, although most have relied exclusively on self-report measures. Despite these methodological problems and the dearth of research from the U.S., the massage therapy profession has grown significantly and massage therapy is increasingly practiced in traditional medical settings, highlighting the need for more rigorous research.

\section{Keywords}

Massage therapy research review

\begin{abstract}
Since a rather extensive massage therapy research review by Field was published in 2014, a significant literature has amassed on massage therapy effects on a variety of conditions that are reviewed here [1]. Empirical studies, systematic reviews and meta-analyses on massage therapy effects were found on a Pubmed search for the years 2013-2016. The studies were evaluated for treatment integrity, sample size and reliability of measures. Of the 159 studies that were initially selected for review, 79 were excluded because the entire article could not be located or the full paper was in a foreign language or the research failed to meet the criteria of being an empirical study (single-arm, randomized controlled waitlist or treatment comparison), a systematic review or a meta-analysis. Qualitative studies, case studies and small sample size studies were excluded. The final selection included 9 single-arm studies, 48 randomized controlled trials, 3 systematic reviews and 5 meta-analyses (see tables).

These were focused on a variety of conditions including in vitro fertilization, prematurity,
\end{abstract}

\footnotetext{
Touch Research Institute. University of Miami/Miller School of Medicine. USA. tfield@med.miami.edu.
} 
full-term newborns, autism, skin conditions including burn scars and surgical sores, pain syndromes including fibromyalgia and arthritis, blood pressure and hypertension, autoimmune conditions including asthma and multiple sclerosis, immune disorders including HIV and breast cancer and aging problems including menopause, Parkinson's and dementia. The empirical studies, systematic reviews and meta-analyses on massage therapy effects on these conditions are summarized here with respect to their primary findings, methodological flaws, potential underlying mechanisms, and suggested future research.

\section{In vitro fertilization (see Table 1 for a listing of these studies and their primary results)}

In vitro fertilization is probably the earliest developmental research involving massage therapy. In an in vitro fertilization study, oscillating vibrations were used prior to the transfer of the embryo [2]. The positive effects included greater pregnancy rates based on urine assays and ongoing pregnancies measured by fetal heartrate and birth rates as well as miscarriage rates. The problem with this study, however, was that only those who were willing to undergo the massage treatment were allocated to the massage therapy group, suggesting a potential self-selection problem. Nonetheless, it is a suggestive finding and one of the only studies on the use of massage therapy as an assisted reproduction technique. And the researchers did control for the participants' ages, hormonal substitution protocols and quality of the transfer of embryos, and no adverse effects were noted for the massage therapy. The authors speculated that the improved embryo implantation may have related to relaxation, reduced stress as well as decreased uterine contractions and possibly increased blood flow to that region.

\section{Reducing prematurity by decreasing prenatal depression (see Table 1)}

We have conducted several studies showing that massage therapy by a therapist and by significant others reduces prenatal depression and in turn increases gestational age and birthweight (see Field 2014 for a review) [1]. In a recent study, massage therapy was compared to yoga, with yoga being considered a form of self-massage [3]. Both groups of prenatally depressed women became less depressed and their newborns were greater gestational age and birthweight. Despite the positive findings for both groups, the effects are confounded by yoga also being a source of social support from other prenatally depressed women in the group sessions. Despite these prenatal therapies, some depressed women unfortunately still experience preterm delivery.

\section{Preterm infants (see Table 1)}

\subsection{Growth measures}

The most frequently reported effects for massage therapy with preterm infants are their greater weight gain and earlier hospital discharge. In a recent study, massage therapy was performed twice daily for two weeks for 15 min per session [4]. In this study, weight gain, height and head circumference were significantly increased. In addition, the frequency of pre-feed gastric residual was decreased and the number of bowel movements was increased 
in the group who received massage therapy. The authors concluded that NICU nurses needed to be trained in massage therapy techniques so that all preterm infants could receive massage therapy.

In another recent study, preterm infants were randomly assigned to 3 different groups including an oil massage group, a non-oil massage group and a control group [5]. Mediumchain triglyceride oil was used for the oil massage group as a nutritional supplement. The mean weight gain on the seventh day of the study was $105 \mathrm{~g}$ for the oil massage group, $52 \mathrm{~g}$ for the non-oil massage group and $54 \mathrm{~g}$ weight loss for the control group. The greater weight gain by the oil massage group could be related to the oil being absorbed by the skin which could contribute to the weight gain as has been reported in other studies and/or it could be related to increased vagal activity leading to greater gastric motility resulting in more efficient food absorption, as we have shown in an earlier study [1].

\subsection{Vagal activity and gastric motility}

The improved gastrointestinal function noted in the oil massage study just described [5] may relate to increased vagal activity leading to greater gastric motility, as has been shown in one of our studies on preterm infants [1]. In a related study on vagal activity, preterm infants were assessed for heart rate variability (vagal activity) after two weeks of twice daily massage therapy sessions [6]. The authors reported that the infants showed increased heart rate variability during caregiving, suggesting increased parasympathetic activity.

\subsection{Natural killer cell activity}

In one of the only studies on immune function of preterm infants following massage therapy, the researchers measured natural killer (NK) cell number and NK cell cytotoxicity (activity) [7]. Although the number of NK cells did not differ between the massage and the standard treatment control groups, the increase in NK cell activity was significantly greater for the massage group, particularly for those who received more than 5 days of therapy. The infants were also heavier and showed greater daily weight gain at the end of the study. The increased natural killer cell activity is consistent with data we have reported in other studies on massage therapy effects on individuals with immune disorders including women with breast cancer, and adolescents and adults with HIV ([1]). It is therefore not surprising that once again the immune measure most affected was NK cell activity. But given the greater NK cell activity, it is surprising that the number of infections did not differ between groups in the preterm infant study inasmuch as NK cells "kill" bacterial, viral and cancer cells. That the length of hospital stay was also not affected is inconsistent with data from many other studies on preterm infants. A meta-analysis, for example, suggested that massaged preemies on average have 5-6 fewer days of neonatal intensive care [8]. In the seventeen studies available for the meta-analysis, the massaged infants showed an average daily weight gain of $5.3 \mathrm{~g}$ and a mean of 5.4 days shorter hospital stay.

\subsection{Developmental follow-up}

Preterm infants have rarely been massaged by their mothers in these studies and they have rarely been followed for their long-term development. A recent study accomplished both having the mothers massage the preterm infants and following them to 12 months corrected 
age [9]. The researchers found that those infants who were massaged had higher cognitive scores at 12 months corrected age, consistent with earlier data we published showing similarly higher mental development scores at 6 months of age for those preemies who were massaged [1]. Unlike this recent follow-up study, we had also shown continuing greater weight gain.

\subsection{Other touch therapies for preterm infants}

Massage has been compared to a few other forms of neonatal stimulation including kangaroo care and exercise. In the kangaroo care versus massage therapy study, both were effective in increasing body weight and both resulted in shorter hospital stays [10]. The two forms of therapy may be equally effective inasmuch as they are both stimulating pressure receptors, with the kangaroo care providing the infant's skin pressure from the mother's or the father's skin and by the infant's skin being moved as the parent walks around, in essence stimulating pressure receptors. Although we did not find any studies on the effects of kangaroo care on the parents, just as massaging others can have positive effects on both the massager and massagee, kangaroo care is likely to have effects on both the parent and infant, given the stimulation of pressure receptors.

"Exercise" or the passive flexing and extending of the preterm infants' limbs (as in the middle portion of our preterm infant massage protocol) has been used to enhance bone formation [1]. The infants who received this "exercise" showed greater bone formation. The mechanism is not clear here, although it may relate to greater growth hormone (IGF-1) following massage therapy [1]. "Exercise" and massage therapy appear to have similar effects on preemie weight gain. However, our comparison between the moderate pressure massage and the exercise conditions suggested that vagal activity mediated the relationship between massage and weight gain and calorie intake mediated the relationship between "exercise" and weight gain [11]. A potential underlying mechanism for both the "exercise" and the massage effects may be the stimulation of pressure receptors.

\subsection{Preterm infants with medical complications}

Most of the preterm massage studies to date have been conducted with infants who are no longer experiencing medical complications and are off the respirator. However, in at least one recent study, infants with respiratory distress syndrome were given massage while still on the respirator [12]. In this study the mean oxygen saturation was improved in those who were massaged with sunflower oil. This suggests that massage therapy can also be used with infants who are being treated with continuous positive airway pressure.

\subsection{Massage for NICU nurses}

One of the main concerns about the neonatal intensive care unit is the stress experienced by the nursing staff. Their stress could be reduced by massaging the infants. As we have noted elsewhere, the massager can benefit as much as the massagee [1]. In that study, elderly volunteers massaged infants and were massaged themselves. Their stress hormones were lower, and they made fewer trips to their doctors after a month of massaging the infants. Although nurses may be too busy to provide massages for infants in the NICU, in at least one study, the nurses themselves were given massages to reduce their job stress [13]. In this 
study, the nurses were massaged for 20 min twice per week for 4 weeks. Two weeks after the intervention began, their overall job stress scores were significantly decreased as compared to the control group.

\section{Full-term infants (see Table 1)}

Massage therapy has rarely been studied with full-term infants. This probably relates to weight gain not being a concern for full-term infants. However, increasing numbers of fullterm newborns are receiving massage as it has been known to reduce irritability and sleep problems which are the most frequent complaints made by parents to pediatricians. In a recent study, healthy newborns were provided massage for $15 \mathrm{~min}$ a day and maternal attachment was assessed [14]. In this study the group who received massage had higher scores on the Maternal Attachment Inventory. In one of our studies, we taught mothers to massage their newborns from the day of birth to the end of the first month on a daily basis and compared a group who were massaged with lotion versus a group who were massaged without lotion versus a non-massage control group [15]. Both the mothers and the infants in the lotion massage group had better sleep patterns than those of the non-lotion massage and non-massage control groups. This could relate to the massage without lotion being less comfortable.

Bilirubin levels have also decreased in full-term healthy newborns following four days of twenty minute massages given twice daily [16]. In a very similar study but by a different group of researchers, bilirubin levels were also lower following four days of massage [17]. Thus, massage therapy may reduce the need for phototherapy or complement the phototherapy that is typically given to jaundiced newborns.

\section{Infants with other conditions}

\subsection{Infants with gastroesophageal reflux disease (GERD)}

Infants with GERD have significant reflux problems. In one study a massage therapist taught mothers to massage their GERD infants [18].

The infants were randomly assigned to either a massage therapy or a non-massage control group and the massages were 30 min twice a week for six weeks. By the end of the study the Cortisol levels of the massage group had significantly decreased while the Cortisol levels in the non-massage control group had increased. These researchers also observed the motherinfant feeding interactions [19]. Both the massaging mothers and their infants had higher feeding interaction scores, with the mothers showing more sensitivity to the infants' cues.

\subsection{Infants with asymmetry from congenital muscular torticollis}

Infants with this condition were given massages and passive stretching for $30 \mathrm{~min}$ three times a week [20] At the end of the study the infants showed less asymmetry as measured by the thickness of the two sternocleidomastoid muscles, head tilt and the Torticollis Overall Assessment. Despite these suggestive data from a large sample of infants, methodological problems include the lack of a control group and the potential confound of the infants receiving passive stretching exercises along with their massages. 


\section{Autism (see Table 1)}

Children with autism spectrum disorder often have sleep problems. In a recent review of the literature eight studies were identified that explored non-behavioral and nonpharmacological approaches to managing sleep problems in these children [21]. Positive outcomes were reported for massage therapy. We noted in an earlier study that when parents massaged their children with autism before bedtime, the children's sleep improved (shorter latency to sleep, longer sleep time and fewer nightwakings) [1].

In another study using mothers as the massagers, the mothers massaged their children with autism for 20 min daily for 3 months followed by no massage for 4 months [22]. Saliva was collected to assay oxytocin (the "love hormone") before and after a session during the treatment and during the control period. During the massage therapy period both the children and their mothers had higher oxytocin levels. Although this after treatment period control condition is unusual, the authors clearly anticipated the disappearance of the oxytocin effect when the massage therapy was withdrawn. Surprisingly, the mothers and children complied with the control condition after already experiencing the positive effects of massage. Lack of compliance is probably the rationale for having waitlist control groups as the typical control condition.

\section{Skin conditions (see Table 2)}

\subsection{Cleft-lip scars}

Massage therapy has been used to reduce several skin problems including cleft-lip scars, burn scars, post-surgery scars and ulcers. In a study on cleft-lip scars, five weeks of massage including kneading and intra-oral massage was applied to individuals with cleft-lip [23]. Following the five-week treatment, the researcher reported increased range of motion and strength and increased symmetry. The problem with this study is that the sample was small and it lacked a control or treatment comparison condition.

\subsection{Burn scars}

In a study on burn scars, the participants received massage therapy and were observed on several measures including scar thickness, melanin and scar elasticity [24]. Following the massage therapy treatment period the massage group showed decreased pain, pruritis and scar tissue. These data are consistent with a study we conducted on children with burns, although our massage was focused on the non-burn areas [1]. The children's anxiety levels were reduced and their pain thresholds were increased so that they tolerated the debridement (skin brushing) better, as evidenced by less distress behavior. Similar effects were noted in our study on burns in adults including anxiety and Cortisol levels decreasing prior to debridement if the skin brushing was preceded by a massage [1]. Over the course of the study, pain and depression were also decreased.

\subsection{Surgical scars}

In a literature review on studies on scar management, the authors located 10 publications on scar massage [25]. The treatment protocols were highly variable, ranging from ten minutes 
twice daily to 30 min twice weekly. Of the 30 surgical scars treated with massage, $90 \%$ had improved appearance or their scores on the Scar Assessment Scale were significantly higher. The problems with the studies reviewed, however, are that the treatment protocols were highly varied and the measures were not standardized or objective.

\subsection{Bed sores}

Massage therapy was widely used until the 1950s to prevent and reduce bed sores or ulcers related to immobility in hospitalized patients. Pressure sores reputedly affect some $10 \%$ of patients in hospitals. Despite this problem a recent review of the literature failed to reveal any randomized controlled trials or even quasi-randomized controlled trials that met inclusion criteria for a meta-analysis [26]. Thus there were no studies eligible for this review and it remains uncertain whether massage therapy prevents bed sores.

\section{Pain syndromes (see Table 3)}

The lion's share of massage therapy research continues to relate to pain, probably because most massage therapy clients are receiving the therapy for pain syndromes. Therefore the massage therapist community is most interested in the research on pain. Since the last review approximately 20 randomized controlled trials have been conducted on pain syndromes including pain from muscle injury, labor pain, foot pain, knee pain, pelvic pain, low back pain, carpal tunnel syndrome pain, shoulder pain, neck pain, fibromyalgia, coronary bypass pain and pain in veterans [1].

\subsection{Muscle pain}

The muscle injury studies have typically involved exertion-induced muscle injury (weight lifting or leg pressing) including one in which participants were assigned to an exertioninduced muscle injury condition or a massage therapy group or an exertion-induced muscle injury plus massage therapy group [27]. The exertion involved a single round of bilateral eccentric exercise using the leg press. The results suggested that massage therapy attenuated the impairment of upper extremity function resulting from the exertion-induced muscle injury. That result might be expected given that the leg press also involves the upper extremity. In a similar study on eccentric exercise, the experimental groups received vibration or massage therapy [28]. Muscle soreness was significantly reduced for the two experimental groups versus the standard treatment control group. Range of motion also significantly increased for the massage versus the other groups.

\subsection{Labor pain}

In a study on labor pain, massage was provided for thirty minutes at each stage of labor, and pain and anxiety were measured [29]. The massage group had lower pain and anxiety levels and shorter duration of labor. These data are consistent with our earlier study in which we had partners provide massage for only the first 15 min each hour of labor and found similar effects including less pain and need for medication and an average of $5 \mathrm{~h}$ less labor [1]. 


\subsection{Foot pain}

In a study on foot pain massage therapy and exercise were compared to ultrasound therapy and exercise [30]. Six treatments were given over a period of $4-6$ weeks in a single-blind randomized trial (single-blind basically being the only way a massage therapy study can be conducted with the researcher collecting the data being blinded). At the end of the treatment period the massage participants had lower pain than the ultrasound participants and they reported greater functional status as measured on the foot and ankle pain assessment.

\subsection{Knee arthritis pain}

Massage therapy has been used in individuals with knee arthritis pain in at least 3 recent randomized controlled studies. In one study, massage therapy and an exercise program were combined and compared to an exercise program alone [31]. Significant improvement was noted for both groups on the pain scale, get up and go test and on the WOMAC index, the variables typically used to test massage therapy effects on knee arthritis pain. In this study, the combined exercise/massage therapy led to greater improvement on these variables than the group that received exercise alone.

In another recent knee osteoarthritis study, Chinese massage (moderate pressure massage) was given three times per week for two weeks [32]. Gait was then evaluated using a motion analysis system by infrared cameras. The patients experienced less pain and stiffness and enhanced function. They also showed increased gait speed and greater step width. However, there was no increase in range of motion.

In contrast to other studies that were focused on the quadriceps muscles, the massage protocol of our study on knee osteoarthritis was focused on the hamstrings as well as the quadriceps [33]. We also used moderate pressure massage. We believe that using moderate pressure and massaging both the hamstrings and the quadriceps may have contributed to our unique finding of increased range of motion. Those two aspects may be necessary, i.e. the moderate pressure massage and the focus on both sets of muscles, although, unfortunately, they were confounded in this study, highlighting the need for a replication study.

\subsection{Pelvic pain}

In a study on pelvic pain a treatment comparison was made between massage and isometric exercises [34]. The treatment lasted eight weeks and the two groups were compared to a notreatment control group. Pain intensity was significantly reduced in both treatment groups, although the reduction was greater in the massage group.

\subsection{Carpal tunnel syndrome}

A study on carpal tunnel syndrome involved the location of trigger points on the hand, and the massage was combined with trigger point therapy [35]. The results suggested a significant reduction in pain as well as an increase in functional activity after two weeks of 30-min massages twice per week. It is not clear that the trigger point therapy added anything given that massage therapy alone has been effective for carpal tunnel syndrome and on the same measures including the Phalen test and the Tinel test [1]. 


\subsection{Hand pain and upper limb pain}

Adults with hand pain have been taught massage so that they could massage their hands whenever they experienced pain, and, in addition, one group was given a topical analgesic to apply following the massage to be compared to a group that did the self-massages but without the topical analgesice [36]. The group that had both the massage and the analgesic had greater grip strength and a greater decrease in hand pain, depressed mood and sleep disturbance.

In a study we conducted on rheumatoid arthritis in the upper limbs, individuals were randomly assigned to one group who received moderate pressure massage or to another group who received light pressure massage [37]. After the first and last sessions, the moderate pressure versus the light pressure group showed greater grip strength and diminished pain. By the end of the study the moderate pressure massage group participants again had a greater decrease in pain and showed greater grip strength and greater range of motion in their wrists and upper joints (elbows and shoulders).

\subsection{Neck pain}

Much of the literature on massage therapy on neck pain is mixed depending on the dose level and the comparison groups, and many of the studies are based on self-report. In a randomized controlled study we conducted, massage therapists provided weekly moderate pressure neck massages and the participants were taught to massage themselves so that they could have daily massage [38]. The massage group showed significant immediate reductions in both self-reported pain and range of motion associated pain and an increase in range of motion on the first and last days of the study. The massage group versus the waitlist control group showed increased range of motion and decreased range of motion associated pain on the last versus the first day. These data suggest that moderate pressure massage may contribute to the effects that can be sustained by self-massage between therapist sessions.

In a review of randomized controlled trials that were identified by literature searches of 5 English and Chinese databases, a meta-analysis was conducted on massage therapies versus inactive therapies for neck and shoulder pain [39]. Their meta-analysis suggested that massage therapy yielded greater pain reduction. The authors recommended that massage therapy be compared with aqua therapies. However, when that comparison was made, massage therapy did not yield better effects for neck pain or shoulder pain. Although these results are consistent with our neck massage therapy versus waitlist control effects just described [38], their meta-analysis results suggest that treatment comparisons are more valid than comparing massage therapy with inactive controls, sham treatments or waitlist controls.

A meta-analysis on massage therapy for neck and shoulder pain by a different group further highlights that point [40]. These authors reported immediate effects of massage therapy versus inactive therapies for the reduction of both neck and shoulder pain. However, when massage therapy was compared to other active therapies, massage therapy did not yield better effects. The effects for active therapies may also derive from the stimulation of pressure receptors by the therapies. 


\subsection{Back pain}

Several back pain studies have appeared in the literature comparing massage therapy to other forms of complementary treatment. The frequent study of back pain massage probably relates to the high incidence of low back pain as compared to other forms of pain, although carpal tunnel pain and smart thumb syndrome pain are becoming increasingly common. In one study, women with chronic low back pain were randomly assigned to massage therapy or physical therapy groups [41]. Stretching exercises were added to both the massage therapy and the physical therapy. The data analysis revealed that the massage therapy participants had a greater decrease in pain intensity and disability than the physical therapy group. These results may relate to physical therapy combined with stretching being more strenuous exercise. However, no group differences were noted for range of motion.

In another treatment comparison study, individuals with low back pain were randomly assigned to a Swedish massage with aromatic ginger oil versus a traditional Thai massage group (30 min sessions twice per week for 5 weeks) [42]. The Swedish massage was more effective than the Thai massage in reducing pain and disability. In this study, range of motion was not measured. These results were surprising given that Thai massage typically involves more pressure stimulation. The ginger oil could have had additive effects, although the use of an aromatic oil in one group and not the other confounds this comparison. Having direct stimulation of the skin versus being massaged fully clothed also confounds the comparison.

In a more matched comparison between structural massage (rocking and stretching) versus relaxation massage (stroking), the groups did not differ on self-reported back pain symptoms [43]. However, once again, range of motion was not measured. In our study comparing massage versus relaxation therapy, the massage group showed increased trunk flexion (touching toes to the point of pain and touching toes to the point of no pain) as well as less self-reported pain, depression, anxiety and sleep disturbance after 5 weeks of twice a week 30-min massages [1]. Our study, however, lacked a measure of compliance. The relaxation therapy participants should have had their sessions at the clinic to ensure compliance.

\subsection{Pain in different joints}

In a systematic review on pain in different joints, the 26 eligible randomized controlled trials included 2165 participants [44]. However, twenty of the trials were considered to be at high risk for bias. The results were somewhat mixed in that massage reduced pain in the shortterm for shoulder pain and osteoarthritis of the knee but not for neck pain or low back pain. However, function was improved in the long-term for the individuals with shoulder pain and knee arthritis as well as low back pain. Several of the studies that were reviewed showed no greater benefits for massage than there were for joint manipulation or acupuncture. These results are perhaps not surprising in that each of those three treatment modalities involves stimulation of pressure receptors. The authors concluded, nonetheless, that the comparisons between massage and active treatments such as joint manipulation need to be replicated. 


\subsection{Fibromyalgia}

In a series of fibromyalgia studies, positive effects have been noted for different types of massage therapies [1]. However, in a meta-analysis study on randomized and nonrandomized trials, Shiatsu decreased pain, fatigue and sleep disturbances while Swedish massage did not improve outcomes [45]. This finding was not surprising inasmuch as Shiatsu versus Swedish massage typically involves moderate pressure. In another metaanalysis study on fibromyalgia, nine randomized controlled trials were included [46]. In this analysis, massage therapy with a duration greater than 5 weeks resulted in decreased pain, anxiety and depression, but no change occurred for sleep disturbances.

The results of the latter meta-analysis are inconsistent with the results of one of our fibromyalgia studies, possibly because we measured sleep activity with actometers rather than relying on self-report and we also measured substance $P$ which causes pain [1]. We found an increase in deep/restorative sleep based on the activity recordings (minimal activity being associated with deep sleep) and a decrease in substance $\mathrm{P}$ across the course of the study. Substance P is released when there is insufficient deep/restorative sleep, and substance $\mathrm{P}$ causes pain.

Another example of sleep being improved following massage therapy comes from a Taiwanese study on insomnia in postpartum women [47]. The massage group received a 20min back massage at the same time each evening for 5 consecutive days and the control group received standard treatment. As assessed by the self-report Pittsburgh Sleep Quality Index, the massage group experienced a greater decrease in sleep disturbances than the control group. The enhancement of sleep may be a mediating variable for pain reduction following massage therapy.

\subsection{Veterans}

In one of the very few studies on massage therapy with veterans, positive effects were reported [48]. The 153 veterans who received massage experienced reduced pain and anxiety following massage. These data, however, were pre-post treatment data without a comparison control or treatment group.

\subsection{Coronary bypass and cardiac surgery}

Massage has also been assessed for its effects on pain following coronary artery bypass and cardiac surgery. In the coronary artery bypass surgery study the patients received massage by a relative who was trained by a nurse [49]. The pain intensity measured by a visual analogue scale was decreased at all time points following massage. In a study on elective cardiac surgery patients, visual analogue scales were again used [50]. In this study, there was a 99\% response rate which seems unusually high. Massage therapy led to a greater reduction in pain, muscle tension and anxiety and increased relaxation as compared to a control group who simply received rest for the same period of time as the massage group. This might not be an optimal control condition given that resting may lead to restlessness in a control group not receiving therapy. A control condition like rest can possibly be a negative experience. 
In an even simpler post-surgery massage study, the therapy group received 15-min hand massages and the control group a simple hand-holding for $15 \mathrm{~min}$ [51]. These were given on 3 occasions within $24 \mathrm{~h}$ after surgery. Pain intensity and muscle tension were decreased for the hand massage but not the hand-holding control group. The authors concluded that this was a low-cost non-pharmacologic intervention. Although "hands-on" comparison groups may be better control groups, the hand-holding group may not have received the skinmoving, stimulation of pressure receptors that the massage group received. The pressure being applied needs to be measured, as can be done with a sound meter placed near the skin of the person being touched/massaged [1].

\section{Blood pressure and hypertension (see Table 4)}

The effects of massage therapy on blood pressure have been studied in a number of different situations/conditions. These include recent studies on coronary care, hospitalization for trauma, prehypertension, essential hypertension and exploring potential underlying mechanisms for the reduction of blood pressure following massage therapy.

\subsection{Coronary care}

In a study on coronary care patients, a randomized controlled trial was conducted [52]. The patients were randomly assigned to a whole body massage group or a control group receiving standard treatment. Decreases occurred for both systolic and diastolic blood pressure and for heart rate and respiratory rate after massage therapy. These decreases may relate to the reduced anxiety following massage. In another study, patients in intensive care were randomly assigned to a massage therapy or a control group and vital signs were taken at hourly intervals [53]. Significant decreases were noted in systolic blood pressure at every time interval (1, 2,3 and $4 \mathrm{~h})$.

In another massage by a family member study, trauma ICU patients received a full body 45 min massage by a family member and the control group received routine care [54]. One hour after the intervention significant differences were noted between the two groups, with the massage group having lower systolic and diastolic blood pressure, respiratory rate and heartrate. Significant group differences were also noted in the arterial blood gas measures including 02 saturation, $\mathrm{PH}$ and $\mathrm{p} 02$. The authors recommended massage by a family member as a routine clinical practice.

These were unusual studies in that the massage was conducted by family members and appeared to have yielded similar effects as other studies that involved massage therapists. However, when a direct comparison was made between nurse and family member massage for coronary patients, Cortisol levels were significantly decreased (by $90 \mathrm{nmol}$ ) in the massage by a nurse group but not in the other group [55].

\subsection{Prehypertension}

This condition has been studied primarily in women. In one study Swedish massage was applied to the face, neck, shoulders and chest for 10-15 min three times a week for ten sessions [56]. The data analysis suggested that both systolic and diastolic blood pressure decreased more in the massage than the standard treatment control group. In another study 
by the same group the sustainability of the massage therapy effects was assessed by taking vital signs at $72 \mathrm{~h}$ after completing the study [57]. At the $72 \mathrm{~h}$ follow-up there were still significant differences between the groups in both systolic and diastolic blood pressure favoring the massage therapy group. These surprising data need to be replicated as it is generally assumed that when stimulation is withdrawn, the effects are not sustained [1].

\subsection{Hypertension}

Although massage is often given to hypertensive patients who have a number of problems including anxiety, depression, elevated stress hormones, headache, vertigo, chronic pain in the back, shoulder and neck regions, typically the primary measures are systolic and diastolic blood pressure. In a study we conducted, high blood pressure symptoms were decreased including systolic and diastolic blood pressure as well as depression and urinary and salivary Cortisol [1].

In a meta-analysis, for another example, the outcome measures were systolic and diastolic blood pressure [58]. In this meta-analysis on 24 studies involving 1962 patients, the methodological quality of the trials was low. Nonetheless, the data tentatively suggested that massage was more effective than anti-hypertensive drugs in lowering systolic and diastolic blood pressure. These data need to be replicated in higher quality trials.

\section{Auto-immune conditions (see Table 5)}

Several auto-immune conditions have been studied for massage therapy effects including asthma, diabetes, dermatitis, and multiple sclerosis [1]. The auto-immune research of the last few years has focused primarily on asthma and multiple sclerosis. These studies are summarized here.

\subsection{Asthma}

In a study on children with asthma, random assignments were made to massage and control groups [59]. Twenty-minute therapy sessions were given by the parents every night for five weeks. At the end of the study the mean forced expiratory flow in the first second (FEV1) was significantly higher in the massage group and although there was no difference in forced vital capacity (FVC) or peak expiratory flow, the FEV1/FVC ratio was significantly improved for the massage therapy group. These data are consistent with those we reported several years earlier except that we also found reductions in FVC and peak expiratory flow [1]. The inconsistencies are surprising inasmuch as the recent study was basically a replication of the Field et al. protocol [1]. Nonetheless, these are more objective, valid measures as opposed to self-report data, and except for clinician differences in administering the measures, the inconsistencies are difficult to interpret. Again, replications are needed especially of those studies that have used gold standard medical measures such as these pulmonary assessments. And, wherever possible the reliability of self-reports needs to be checked against the more objective laboratory measures. 


\subsection{Multiple sclerosis}

In studies on multiple sclerosis, self-reports again were the most common measures, not unlike studies on the other conditions already discussed. In the first of these, the Multiple Sclerosis Efficacy Survey was used post massage therapy and at a 4-week and 8-week follow-up period [60]. The massage therapy group showed significantly more improvement on this survey than the waitlist control group at mid-treatment, end of treatment and at the 4week follow-up period but not at the 8-week follow-up, surprisingly suggesting that the positive effects of the massage were sustained for as long as four weeks. In another study, exercise capacity and lung function were added to the self-report measures and the more objective 6-min walk test was administered [61]. Following a Swedish massage for 4 weeks there were no changes on these measures, although the participants reported improvement in quality of life in their written comments. Thus, these two studies have inconsistent findings, with the first study on therapeutic massage yielding positive change and the second study using Swedish massage reporting no change. This inconsistency may have related to the first study using therapeutic massage which typically involves more moderate pressure and/or it may have related to the more positive subjective self-report used in the first study versus the more objective walking measure used in the second study. Multiple subjective and objective, self-report and laboratory measures are needed to resolve these inconsistencies.

Another inconsistency is suggested by the positive changes reported for individuals with multiple sclerosis in another Swedish massage study [62]. In this study the patients were randomly assigned to four groups including massage therapy, exercise therapy, massage therapy combined with exercise therapy and a control group. The massage was provided for 15 sessions over 5 weeks and consisted of Swedish massage techniques. The exercise group was given a combination of strength, stretch and balance exercises. The results suggested that the massage therapy group experienced a greater reduction in pain and greater improvement in balance and the walking assessment than the other groups. The patients in the combined massage and exercise group showed greater improvement in balance than the exercise group. It is not clear why the massage group experienced greater improvement than the massage plus exercise group. The exercise may have exhausted the patients and thereby attenuated the positive effects of massage for these individuals with multiple sclerosis.

The inconsistent findings between the two Swedish massage therapy studies are difficult to interpret. It is not clear whether the Swedish massage techniques used in these two studies were different. However, it is striking that the same objective measures yielded different results for the two studies. The null finding study may have been using light pressure massage techniques and/or it may have sampled more severe multiple sclerosis patients. The greater dose of massage in the positive effects study ( 5 weeks versus 4 weeks) may have made the difference. Just as for the other conditions, the multiple sclerosis studies have the problem that different types of massage were used and different measures were assessed and most measures were by self-report.

\section{Immune disorders (see Table 5)}

Immune conditions that have been studied since our last review on massage therapy research [1] have included HIV and cancer. The HIV studies summarized here include a study on 
HIV-exposed infants and another on HIV in adults. Breast cancer has been the most frequently researched cancer in the recent studies on massage therapy.

\subsection{HIV}

In the study on HIV-exposed infants, the mothers with HIV were taught to massage their infants which they did between 6 weeks when the study started and 9 months when the study ended [63]. Despite the massage group mothers having higher levels of maternal "mental pain", their infants scored significantly higher on scales of mental development and hearing and speech at 9 months. Even though the CD4 cell count (the critical index of HIV severity) was measured in the mothers, there was no report on that measure or any measure of the effects of the infant massage on the mothers themselves. This is surprising given that the mothers presumably benefited from massaging their infants. As has been documented in at least a few studies, and as already discussed, massaging others has very similar stresslowering (stress hormone-lowering) effects on the massager as well as the massagee [1]. In one of those studies elderly participants were noted to have lower norepinephrine levels and fewer trips to their physicians following a period of massaging infants [1].

In a study on HIV adults with a major depressive disorder, one group received Swedish massage and the second group received touch alone [64]. The massage was received for one hour twice per week. For the touch group, the massage therapist placed both hands on the patients' body with slight pressure but no movement. The massage significantly reduced the depression by the fourth week and continuing at the sixth and eighth weeks in contrast to there being no effects of the touch or the no-touch conditions. This is one of the rare studies that compared massage with simple touch. It is not surprising that the Swedish massage subjects experienced a greater decrease in depression than the touch alone group given that the touch group only involved hands-on and no movement of the skin which would be necessary to achieve positive effects. Once again, however, it is surprising that although these HIV studies measured immune function to ensure baseline equivalence of the groups, they have not reported the effects of the massage on immune function.

In massage studies by our group on adolescents with HIV and adults with HIV [1] significant decreases in Cortisol and increased natural killer cell number as well as natural killer cell activity were noted. In the study on HIV adolescents CD4 count also increased [1]. These data suggest a slowed progression of the disease both because natural killer cells "kill" viral cells, and CD4 cells are the cells killed by the HIV virus, so the increases in these immune measures are clinically significant.

\subsection{Cancer}

The cancer studies also used self-report measures in most cases. In one study on children with cancer, massage therapy was used to reduce anxiety and pain associated with bone marrow aspiration [65]. In this study, the children used visual analogue scales to report their pain and anxiety levels. Both these levels were significantly reduced in the massage therapy group. However, when the pretest and posttest scores were compared, no significant differences were noted between the massage and control groups. 
Among the most negative side effects of chemotherapy are nausea and vomiting. In another pediatric cancer study, the effects of massage therapy on chemotherapy associated nausea and vomiting were measured [66]. The massage group children received 20 min massages $24 \mathrm{~h}$ before and $24 \mathrm{~h}$ after chemotherapy and they were given visual analogue scales for pain and intensity. Time and length of nausea and vomiting were also recorded. The results suggested that the pain severity was less for the massage group and the children in that group also had fewer bouts of vomiting. Massage therapy and ginger have also been identified as a therapeutic combination for adults' chemotherapy-induced nausea and vomiting [67].

In studies on women with breast cancer, the quality of sleep, the levels of depression, stress catecholamines, and immune cells including cytokines (Thl/Th2 immune balance) and natural killer cells have been measured following massage therapy. In a sleep study, the authors noted that insomnia was one of the most common problems following breast cancer [68]. In this study women with breast cancer were randomly assigned to a medical massage therapy group or a control group who received usual medical care. The results revealed prepost intervention differences in immune scores and quality of sleep in the massage group based on the Pittsburgh Sleep Quality Index. Although medical massage usually involves moderate pressure so the effects reported here might be expected, it is unclear that the statistics they used involved an appropriate comparison between groups as a group by repeated measures analysis or simply pre-post comparisons for the separate groups.

In an immune study on breast cancer patients, the women were given two 30-min massages per week for five weeks [69]. The results suggested that depression and anxious depression were significantly reduced after the massage compared to the control condition. There was also an increase in Th1 over time for the massage group. That shift is significant in that the $\mathrm{Thl} / \mathrm{Th} 2$ ratio is an important index of immune function with lower production of cytokines (pro-inflammatory cells associated with Th2) being a positive change. In another breast cancer study we reported reduced depression and increased dopamine and serotonin (both activating neurotransmitters) as well as increased natural killer cell number and lymphocytes [1]. The increased dopamine and serotonin may have mediated both the decrease in depression and the increased immune cells.

In a study on reconstruction after mastectomy for breast cancer, patients were randomly assigned to either a massage therapy group or a massage plus meditation group [70]. There were no additive effects of meditation on any of the self-report stress, insomnia, fatigue or pain measures. Although it is generally methodologically appropriate to assess the effects of two therapies combined versus one alone, the addition of meditation may have placed demands on the participants that were new to them, resulting in performance stress. Also, the comparisons can be confounded by the additional time demands on the participants when two therapies are combined.

Massage therapy effects have also been studied in patients with leukemia, but unfortunately only using self-report measures including stress and quality of life scales [71]. While the massage therapy group showed a significant decrease in stress and improvement in quality of life, the relatively small sample limits the generalizability of the data. 
In a meta-analysis on the effects of massage therapy on cancer in general, cancer-associated pain was noted to be one of the most common complaints [72]. Nine high quality studies were included in this meta-analysis. Massage therapy was noted to significantly reduce cancer pain as compared to no massage control conditions. Massage was effective especially for surgery-related pain, and among the various types of massage, foot reflexology was the most effective. It may have been the most effective as it typically involves the application of moderate pressure and the movement of skin perhaps moreso than Swedish massage.

\section{Aging (see Table 6)}

Very few studies have focused on the effects of massage therapy on aging conditions. Recent studies on its effects on postmenopausal women, on Parkinson's and on dementia are summarized here.

\subsection{Postmenopausal women}

In a study on postmenopausal women 30-min sessions for four weeks of massage were provided with aromatherapy or with odorless oil [73]. These groups were compared to a non-massage group on measures of psychological symptoms on the postmenopausal scale. The aromatherapy-massage group showed the greatest decreases in psychological symptoms. This is not surprising since the literature has suggested that adding an aroma oil to the massage has additive effects and the aroma oils themselves have been noted to alter brainwaves in the direction of relaxation and reduced heartrate [1].

In another study on postmenopausal women, biochemical markers of bone formation were the primary measures [74]. In this study women were randomized to a two-hour session of Thai massage twice a week for four weeks and compared to a waitlist control group. The results suggested that serum P1NP (a primary biochemical bone formation marker) increased significantly after Thai massage while there was no change for the control group. The Thai massage was particularly effective for women who were older and had a smaller body build. The results of the study are interesting in that they suggest that even after a short period of four weeks, bone formation can occur following Thai massage. Inasmuch as Thai massage involves significant moving of the skin and moving the limbs, even more stimulation of pressure receptors may occur with this type of massage, although Thai massage is rarely studied.

\subsection{Parkinson's}

In a study on Parkinson's, Amma massage therapy was used to alleviate physical symptoms [75]. This study basically assessed the effects of one forty-minute Amma massage session involving upper and lower limb exercises as compared to a control group. After only one session, the analogue scale scores were lower for muscle stiffness, movement difficulties, pain and fatigue. On the more objective measures, gait speed was significantly faster, stride length was lengthened and shoulder flexion and abduction were improved. After several sessions the authors found improvements on the same measures. These effects are interesting in that Amma massage, like Thai massage, is given through clothing, making it more accessible to patients like those with Parkinson's. These data are consistent with our study on 
moderate pressure massage in terms of improved functioning in patients with Parkinson's [1]. We also noted a decrease in norepinephrine and epinephrine (stress neurotransmitters) which could mediate the physical symptoms associated with Parkinson's.

\subsection{Dementia}

In a study on elderly patients with dementia, the effects of massage therapy and ear acupuncture were compared with a control group [76]. Behavior alterations including sleep disturbance, eating and compliance were recorded during the three months of intervention. Positive effects were noted for massage and ear acupuncture when compared to the control group and they were persistent for two months after completing the treatment. The long-term effects are surprising given that continued stimulation of pressure receptors would be presumably needed for the effects to continue. This study also suggests that massage therapy and acupuncture might have similar underlying mechanisms, for example, that they involve the stimulation of pressure receptors, increased vagal activity and a reduction of Cortisol.

\subsection{Potential underlying mechanisms}

In a study that examined the underlying mechanisms for the effects of massage, for example, on blood pressure, a thematic analysis was conducted on 27 studies that considered the effects of massage therapy [77]. The author identified several potential underlying pathways for the relationship between massage therapy and the reduction of blood pressure. Just as we have previously noted [1], this author also suggested that massage therapy has effects via increased parasympathetic activity and decreased Cortisol or improved functioning of the hypothalamic-pituitary-adrenal-cortical (HPAC) system.

Examples have been seen in the literature on how parasympathetic activity can be increased by massage therapy, for example, in children [78]. In this study, parasympathetic activity increased significantly in children receiving foot and hand massage on a pediatric intensive care unit. Repeated sessions suggested that parasympathetic activity peaked after the second session and remained stable for the remaining sessions. Another study documented increased parasympathetic activity following a single short session $(10 \mathrm{~min})$ of head massage [79]. The head massage also reduced heartrate.

An example of the effects of massage on the HPAC system or the levels of Cortisol can be seen in a study that involved the patients' companions giving them full body massages [80]. In this randomized controlled trial on 60 patients admitted to intensive care those who received massage had significantly lower Cortisol levels. Again, this was an unusual study given that the massages were provided by the patients' companions. However, the reduction in Cortisol was not surprising given that Cortisol levels have significantly decreased by an average of $31 \%$ across our massage therapy studies [1].

\section{Limitations of the therapy protocols}

Massage therapy has typically been compared to a standard treatment control group. As massage therapy is increasingly noted to be therapeutic, an ethical question is whether a control group can be denied therapy. Increasing numbers of studies are therefore using waitlist control groups or comparison treatment groups. The waitlist control group would 
receive the same massage therapy at the end of the waitlist period. And treatment comparison groups would be given a similar therapy. The problem has been matching therapy protocols so they are not confounded by variables such as length of massage, duration of treatment period, and pressure applied. Some of the comparisons have been between different massage techniques, for example, Swedish versus Thai massage. The findings from those comparisons have often been mixed, with some studies suggesting that Swedish massage was more effective than Thai massage and other studies yielding the opposite effects. These are very different therapies with the Thai massage being fully clothed and the other not. In addition, Thai massage is typically a longer session than Swedish massage and Thai massage characteristically involves more movement of the joints and the skin. So these comparisons have been confounded by a number of basic differences between the protocols. Massage has also been compared to simple touch and is more effective probably because of the movement of the skin. And, massage has generally yielded better results than exercise, especially with individuals like those with multiple sclerosis who might find exercise too exhausting.

When a therapy is added to massage therapy it sometimes has additive effects. For example, adding aroma to massage oils has been effective. Other therapies have had no additive effect, for example, exercise, possibly because it was too exhausting. The added therapy needs to be assessed as a group on its own. So, comparisons, for example, would be made between a massage group, an exercise group and group that receives both massage and exercise. When exercise is added to massage, that combination may attenuate the positive effects of massage, again because an exhausting modality is being added to a relaxing modality. In contrast, other active therapies like yoga and tai chi and other forms of exercise may have positive effects as they are not exhausting and they involve stimulation of pressure receptors much like massage.

\section{Limitations of the measures}

The massage research reviewed here as compared to that reviewed in 2014 [1] has typically involved self-report measures even though measurement technology has become increasingly sophisticated. For example, in the earlier studies on breast cancer, immune measures were the primary measures, but in some of the recent studies on breast cancer, immune measures were not even reported. Gold standard medical measures have been used in some studies as, for example, blood pressure measures in hypertension studies, CD4 cells in HIV studies and range of motion measures in arthritis studies, but most of the studies reviewed in this paper relied on self-report measures. It is not clear why this focus on selfreport measures has occurred as the reliability of self-report measures has been questionable. Their excessive use may in part relate to limited funding in recent years in this country and to limited funding in general in Asian countries where most of the massage therapy studies have been conducted. Ideally, research protocols would include psychological, physical, physiological and biochemical measures to document multivariable effects. 


\section{Limitations of the conditions studied}

Pain syndromes continue to receive the lion's share of the research, probably because the most frequent massage therapy clients are those with pain syndromes, highlighting the importance of that research. Massage therapy research has also increasingly focused on hypertension and breast cancer. In contrast, some conditions that are also very prevalent in this country have not been assessed for massage therapy effects including obesity and diabetes (both conditions being NIH funding priorities) and drug use (a NIDA funding priority). Even though the growth of the massage therapy industry in the U.S. has been exponential, despite very limited insurance coverage for that therapy, relatively few researchers are studying massage therapy. Despite these problems with the literature, massage therapy is now considered more than just a complementary therapy and has been making inroads into more traditional medical settings.

\section{Summary}

In this review, massage therapy has been shown to have beneficial effects on many different groups and conditions including prenatal depression, preterm infants, full-term infants, autism, skin conditions, pain syndromes including arthritis and fibromyalgia, hypertension, autoimmune conditions including asthma and multiple sclerosis, immune conditions including HIV and breast cancer and aging problems including Parkinson's and dementia. Although many of the studies have involved comparisons between massage therapy and standard treatment control groups, several have compared different forms of massage (e.g. Swedish versus Thai massage), and different active therapies such as massage versus exercise. Typically, the massage therapy groups have experienced more positive effects than the control or comparison groups, potentially because massage involves the stimulation of pressure receptors leading to enhanced vagal activity and reduced Cortisol levels. Some of the researchers have employed physical, physiological and biochemical measures, although most of them have relied exclusively on self-report measures. Despite these methodological problems and the dearth of research from the U.S., the massage therapy profession has grown significantly and massage therapy is increasingly practiced in traditional medical settings, highlighting the need for more rigorous studies.

\section{Acknowledgments}

I would like to thank my collaborators, the individuals who participated in these studies and the research associates who assisted us. This research was supported by funding from Johnson and Johnson and Massage Envy to the Touch Research Institute. Correspondence and requests for reprints should be sent to Tiffany Field, Ph.D., Touch Research Institute, University of Miami Medical School, P.O Box 016820, Miami, Fl 33101. tfield@med.miami.edu.

\section{References}

1. Field T. Massage therapy research review, Complement. Ther Clin Pract. 2014; 20:224-229.

2. Okhowat J, Murtinger M, Schuff M, Wogatzy J, Spitzer D, Vanderzwalmen P, Wirleitner B, Zech $\mathrm{NH}$. Massage therapy improves in vitro fertilization outcome in patients undergoing blastocyst transfer in a cryo-cycle, Altern. Ther Health Med. 2015; 21:16-22. 
3. Field T, Diego M, Hernandez-Reif M, Medina L, Delgado J, Hernandez A. Yoga and massage therapy reduce prenatal depression and prematurity. J Bodyw Mov Ther. 2012; 16:204-209. [PubMed: 22464118]

4. Choi H, Kim SJ, Oh J, Lee MN, Kim S, Kang KA. The effects of massage therapy on physical growth and gastrointestinal function in premature infants: a pilot study. J Child Health Care. 2015 Epub ahead of print.

5. Saeadi R, Ghorbani Z, Shapouri Moghaddam A. The effect of massage with medium-chain triglyceride oil on weight gain in premature neonates. Acta Med Iran. 2015; 53:134-138. [PubMed: 25725185]

6. Smith SL, Haley S, Slater H, Moyer-Mileur LJ. Heart rate variability during caregiving and sleep after massage therapy in preterm infants. Early Hum Dev. 2013; 89:525-529. [PubMed: 23361061]

7. Ang JY, Lua JL, Mathur A, Thomas R, Asmar BI, Savasan S, Buck S, Long M, Shankaran S. A randomized placebo-controlled trial of massage therapy on the immune system of preterm infants. Pediatrics. 2012; 130:1549-1558.

8. Wang L, He JL, Zhang XH. The efficacy of massage on preterm infants: a meta-analysis. Am J Perinatol. 2013; 30:731-738. [PubMed: 23322391]

9. Abdallah B, Badr LK, Hawwari M. The efficacy of massage on short term and long term outcomes in preterm infants. Infant Behav Dev. 2013; 36:662-669. [PubMed: 23932956]

10. Rangey PS, Sheth M. Comparative effect of massage therapy versus kangaroo mother care on body weight and length of hospital stay in low birth weight preterm infants. Int J Pediatr. 2014 Epub ahead of print.

11. Diego M, Field T, Hernandez-Reif M. Preterm infant weight gain is increased by massage therapy and exercise via different underlying mechanisms. Early Hum Dev. 2014; 90:137-140. [PubMed: 24480603]

12. Valizadeh S, Hossieini MB, Asghari Jafarabadi M, Ajoodanian N. The effects of massage with coconut and sunilower oils on oxygen saturation of premature infants with respiratory distress syndrome treated with nasal continuous positive airway pressure. J Caring Sci. 2012; 1:191-199. [PubMed: 25276695]

13. Nazari F, Mirzamohamadi M, Yousefi H. The effect of massage therapy on occupational stress of intensive care unit nurses. Iran J Nurs Midwifery Res. 2015; 20:508-515. [PubMed: 26257809]

14. Curol A, Polat S. The effects of baby massage on attachment between mother and their infants. Asian Nurs Res Korean Soc Nurs Sci. 2012; 6:35-41. [PubMed: 25030689]

15. Field T, Gonzalez C, Diego M, Mindell J. Mothers' massaging their newborns with lotion versus no lotion enhances mothers' and newborns' sleep. 2016 (in press).

16. Dalili H, Sheikhi S, Shariat M, Haghnazanan E. Effects of baby massage on neonatal jaundice in healthy Iranian infants: a pilot study. Infant Behav Dev. 2015; 42:22-26. [PubMed: 26646074]

17. Basiri-Moghadam M, Basiri-Moghadam K, Kianmehr M, Jam S. The effect of massage on neonatal jaundice in stable preterm newborn infants: a randomized controlled trial. J PakMed Assoc. 2015; 65:602-606.

18. Neu M, Pan Z, Workman R, Marcheggianni-Howard C, Furuta G, Laudenslager ML. Benefits of massage therapy for infants with symptoms of gastroesophageal reflux disease. Biol Res Nurs. 2014; 16:387-397. [PubMed: 24379449]

19. Neu M, Schmiege SJ, Fehringer K, Workman R, Marcheggianni-Howard C, Furuta GT. Interactions during feeding with mothers and their infants with symptoms of gastroesophageal reflux. J Altern Complement Med. 2014; 20:493-499. [PubMed: 24742255]

20. Lee K, Chung E, Koh S, Lee BH. Outcomes of asymmetry in infants with congenital muscular torticollis. J Phys Ther Sci. 2015; 27:461-464. [PubMed: 25729191]

21. McLay LK, France K. Empirical research evaluating non-traditional approaches to managing sleep problems in children with autism. Dev Neurorehabil. 2016; 19:123-134. [PubMed: 24724691]

22. Tsuji S, Yuhi T, Furuhara K, Ohta S, Shimizu Y, Higashida H. Salivary oxytocin concentrations in seven boys with autism spectrum disorder received massage from their mothers: a pilot study. Front Psychiatry. 2015; 6:58. Epub ahead of print. [PubMed: 25954210]

23. McKay E. Assessing the effectiveness of massage therapy for bilateral cleft lip reconstruction scars. Int J Ther Massage Bodyw. 2014; 7:3-9. 
24. Cho YS, Jeon JH, Hong A, Yang HT, Yim H, Cho YS, Kim DH, Hur J, Kim JH, Chun W, Lee BC, Seo $\mathrm{CH}$. The effect of burn rehabilitation massage therapy on hypertrophic scar after a burn: a randomized control trial. Burns. 2014; 40:1513-1520. [PubMed: 24630820]

25. Shin TM, Bordeaux JS. The role of massage in scar management:: a literature review. Dermatol Surg. 2012; 38:414-423. [PubMed: 22093081]

26. Zhang Q, Sun Z, Yue J. Massage for preventing pressure ulcers. Cochrane Database Syst Rev. 2015:6. Epub ahead of print.

27. Franklin NC, Ali MM, Robinson AT, Norkeviciute E, Phillips SA. Massage therapy restores peripheral vascular function after exertion. Arch Phys Med Rehabil. 2014; 95:1127-1134. [PubMed: 24583315]

28. Imtiyaz S, Vegar Z, Shareef MY. To compare the effect of vibration therapy and massage in prevention of delayed onset muscle soreness (DOMS). J Clin Diagn Res. 2014; 8:133-136.

29. Mortazavi DH, Khaki S, Moradi R, Heidari K, Vasegh Rahimparvar SF. Effects of massage therapy and presence of attendant on pain, anxiety and satisfaction during labor. Arch Gynecol Obstet. 2012; 286:19-23. [PubMed: 22271239]

30. Saban B, Deutscher D, Ziv T. Deep massage to posterior calf muscles in combination with neural mobilization exercises as a treatment for heel pain: a pilot randomized clinical trial. Man Ther. 2014; 19:102-108. [PubMed: 24090993]

31. Cortes Godoy V, Gallego Izquierdo T, Lazaro Navas I, Pecos Martin D. Effectiveness of massage therapy as co-adjuvant treatment to exercise in osteoarthritis of the knee: a randomized control trial. J Back Musculoskelet Rehabil. 2014; 27:521-529. [PubMed: 24867903]

32. Qingguang Z, Min F, Li G, Shuyun J, Wuquan S, Yong L. Gait analysis of patients with knee osteoarthritis before and after Chinese massage treatment. J Tradit Chin Med. 2015; 35:411-416. [PubMed: 26427110]

33. Field T, Diego M, Gonzalez G, Funk CG. Knee arthritis pain is reduced and range of motion is increased following moderate pressure massage therapy, Complement. Ther Clin Pract. 2015; 21:233-237.

34. Azima S, Bakhshayesh HR, Kaviani M, Abbasnia K, Sayadi M. Comparison of te effect of massage therapy and isometric exercises on primary dysmenorrhea: a randomized controlled clinical trial. J Pediatr Adolesc Gynecol. 2015; 28:486-491. [PubMed: 26272447]

35. Elliott R, Burkett B. Massage therapy as an effective treatment for carpal tunnel syndrome. J Bodyw Mov Ther. 2013; 17:332-338. [PubMed: 23768278]

36. Field T, Diego M, Solien-Wolfe L. Massage therapy plus topical analgesic is more effective than massage alone for hand arthritis pain. J Bodyw Mov Ther. 2013; 2014(18):322-325.

37. Field T, Diego M, Delgado J, Garcia D, Funk CG. Rheumatoid arthritis in upper limbs benefits from moderate pressure massage therapy. Complement Ther Clin Pract. 2013; 19:101-103. [PubMed: 23561068]

38. Field T, Diego M, Gonzalez G, Funk CG. Neck arthritis pain is reduced and range of motion is increased by massage therapy. Complement Ther Clin Pract. 2014; 20:219-223. [PubMed: 25444416]

39. Cheng YH, Huang GC. Efficacy of massage therapy on pain and dysfunction in patients with neck pain: a systematic review and meta-analysis. Evid Based Complement Altern Med. 2014 Epub ahead of print.

40. Kong LJ, Zhan HS, Cheng YW, Yuan WA, Chen B, Fang M. Massage therapy for neck a 2013. Epub ahead of print.

41. Kamali F, Panahi F, Ebrahimi S, Abbasi L. Comparison between massage and routine physical therapy in women with sub-acute and chronic nonspecific low back pain. J Back Musculoskelet Rehabil. 2014 Epub ahead of print.

42. Sritoomma N, Moyle W, Cooke M, O'Dwyer S. The effectiveness of Swedish massage with aromatic ginger oil in treating chronic low back pain in older adults: a randomized controlled trial. Complement Ther Med. 2014; 22:26-33. [PubMed: 24559813]

43. Cherkin DC, Sherman Kj, Kahn J, Wellman R, Cook AJ, Johnson E, Erro J, Delaney K, Deyo RA. A comparison of the effects of 2 types of massage and usual care on chronic low back pain: a randomized, controlled trial. Ann Intern Med. 2011; 155:1-9. [PubMed: 21727288] 
44. Bervoets DC, Luijsterburg PA, Alessie JJ, Buijs MJ, Verhagen AP. Massage therapy has short-term benefits for people with common musculoskeletal disorders compared to no treatment: a systematic review. J Physiother. 2015; 61:106-116. [PubMed: 26093806]

45. Yuan SL, Matsutani LA, Marques AP. Effectiveness of different styles of massage therapy on fibromyalgia: a systematic review and meta-analysis. Man Ther. 2015; 20:257-264. [PubMed: 25457196]

46. Li YH, Wang FY, Feng CQ, Yang XF, Sun YH. Massage therapy for fibromyalgia: a systematic review and meta-analysis of randomized controlled trials. PLoS One. 2014:9. Epub ahead of print.

47. Ko YL, Lee HJ. Randomized controlled trial of the effectiveness of using back massage to improve sleep quality among Taiwanese insomnia postpartum women. Midwifery. 2014; 30:60-64. [PubMed: 23410501]

48. Mithchinson A, Fletcher CE, Kim HM, Montagnin M, Hinshaw DB. Integrating massage therapy within the palliative care of veterans with advanced illnesses: an outcome study. Am J Hosp Palliat Care. 2014; 31:6-12. [PubMed: 23423774]

49. Najafi SS, Rast F, Momennasab M, Ghazinoor M, Dehghanrad F, Mousavizadeh SA. The effect of massage therapy by patients' companions on severity of pain in the patients undergoing post coronary artery bypass graft surgery: a single-blind randomized clinical trial. Int J Community Based Nurs Midwifery. 2014; 2:128-135. [PubMed: 25349854]

50. Braun LA, Stanguts C, Casanelia L, Spitzer O, Paul E, Vardaxis NJ, Rosenfeldt F. Massage therapy for cardiac surgery patients: a randomized trial. J Thorac Cardiovasc Surg. 2012; 144:1453-1459. [PubMed: 22964355]

51. Boitor M, Martorella G, Arbour C, Michaud C, Gelinas C. Evaluation of the preliminary effectiveness of hand massage therapy on postoperative pain of adults in the intensive care unit after cardiac surgery: a pilot randomized controlled trial. Pain Manag Nurs. 2015; 16:354-366. [PubMed: 26025795]

52. Adib-Hajbaghery M, Abasi A, Rajabi-Beheshtabad R. Whole body massage for reducing anxiety and stabilizing vital signs of patients in cardiac care unit. Med J Islam Repub Iran. 2014; 28:47. Epub ahead of print. [PubMed: 25405113]

53. Vahedian-Azimi A, Ebadi A, Jafarabadi MA, Saadat S, Ahmadi F. Effect of massage therapy on vital signs and GCS scores of ICU patients: a randomized controlled clinical trial. Trauma Mon. 2014:19.

54. Hatefi M, Jaafarpour M, Khani A, Khajavikhan J, Kokhazade T. The effect of whole body massage on the process and physiological outcome of trauma ICU patients: a double-blind randomized clinical trial. J Clin Diagn Res. 2015:9. Epub ahead of print.

55. Adib-Hajbaghery M, Rajabi-Beheshtabad R, Ardjmand A. Comparing the effect of whole body massage by a specialist nurse and patients' relatives on blood Cortisol level in coronary patients. ARYA Atheroscler. 2015; 11:126-132. [PubMed: 26405441]

56. Moeini M, Givi M, Chasempour Z, Sadeghi M. The effect of massage therapy on blood pressure of women with prehypertension. Iran J Nurs Midwifery Res. 2011; 16:61-70. [PubMed: 22039381]

57. Givi M. Durability of effect of massage therapy on blood pressure. Int J Prev Med. 2013:45114516.

58. Xiong XJ, Li SJ, Zhang YQ. Massage therapy for essential hypertension: a systematic review. J Hum Hypertens. 2014 Epub ahead of print.

59. Fattah MA, Hamdy B. Pulmonary functions of children with asthma improve following massage therapy. J Altern Complement Med. 2011; 17:1065-1068. [PubMed: 22087616]

60. Finch P, Bessonnette S. A pragmatic investigation into the effects of massage therapy on the self efficacy of multiple sclerosis clients. J Bodyw Mov Ther. 2014; 18:11-16. [PubMed: 24411144]

61. Schroeder B, Doig J, Premkumar K. The effects of massage therapy on multiple sclerosis patients' quality of life and leg function. Evid Based Complement Altern Med. 2014 Epub ahead of print.

62. Negahban H, Rezaie S, Goharpey S. Massage therapy and exercise therapy in patients with multiple sclerosis: a randomized controlled pilot study. Clin Rehabil. 2013; 27:1126-1136. [PubMed: 23828184] 
63. Perez EM, Carrara H, Bourne L, Berg A, Swanevelder S, Hendricks MK. Massage therapy improves the development of HIV-exposed infants living in a low socio-economic, periurban community of South Africa. Infant Behav Dev. 2015; 38:135-146. [PubMed: 25645599]

64. Poland RE, Gertsik L, Favreau JT, Smith SI, Mirocha JM, Rao U, Daar ES. Open-label, randomized parallel-group controlled clinical trial of massage for treatment of depression in HIVinfected subjects. J Altern Complement Med. 2013; 19:334-340. [PubMed: 23098696]

65. Celebioglu A, Gurol A, Yildirim ZK, Buyukavci M. Effects of massage therapy on pain and anxiety arising from intraethecal therapy or bone marrow aspiration in children with cancer. Int $\mathrm{J}$ Nurs Pract. 2015; 21:797-804. [PubMed: 24689780]

66. Mazuim S, Chaharsoughi NT, Banihashem A, Vashani HB. The effect of massage therapy on chemotherapy-induced nausea and vomiting in pediatric cancer. Iran J Nurs Midwifery Res. 2013; 18:280-284. [PubMed: 24403922]

67. Sheikhi MA, Ebadi A, Talaeizadeh A, Rahmani H. Alternative methods to treat nausea and vomiting from cancer chemotherapy. Chemother Res Pract. 2015 Epub ahead of print.

68. Kashani F, Kashani P. The effect of massage therapy on the quality of sleep in breast cancer patients. Iran J Nurs Midwifery Res. 2014; 19:113-118. [PubMed: 24834078]

69. Krohn M, Listing M, Tiahjono G, Reisshauer A, Peters E, Klapp BF, Rauchfuss M. Depression, mood, stress, and Thl/Th2 immune balance in primary breast cancer patients undergoing classical massage therapy. Support Care Cancer. 2011; 19:1303-1311. [PubMed: 20644965]

70. Dion LJ, Engen DJ, Lemaine V, Lawson Dk, Brock CG, Thomley BS, Cha SS, Sood A, Bauer BA, Wahner-Roedler DL. Massage therapy alone and in combination with meditation for breast cancerpatients undergoing autologous tissue reconstruction: a randomized pilot study. Complement Ther Clin Pract. 2015 Epub ahead of print.

71. Taylor AG, Snyder AE, Anderson JG, Densmore JJ, Bourguignon C. Gentle massage improves disease and treatment related symptoms in patients with acute myelogenous leukemia. J Clin Trials. 2014:4. Epub ahead of print.

72. Lee SH, Kim jY, Yeo S, Kim SH, Lim S. Meta-analysis of massage therapy on cancer pain. Integr Cancer Ther. 2015; 14:297-304. [PubMed: 25784669]

73. Taavoni S, Darsareh F, Joolaee S, Haghani H. The effect of aromatherapy massage on the psychological symptoms of postmenopausal Iranian women. Complement Ther Med. 2013; 21:158-163. [PubMed: 23642946]

74. Saetung S, Chailurkit LO, Ongphiphadhanakul B. Thai traditional massage increases biochemical markers of bone formation in postmenopausal women: a randomized crossover trial, BMC Complement. Altern Med. 2013; 13:69. Epub ahead of print.

75. Donoyama N, Suoh S, Ohkoshi N. Effectiveness of Anma massage therapy in alleviating physical symptoms in outpatients with Parkinson's disease: a before-after study. Complement Ther Clin Pract. 2014 Epub ahead of print.

76. Rodriguez-Mansilla J, Gonzalez-Lopez-Arza MV, Varela-Donoso E, Montanero-Fernandez J, Jimenez-Palomares M, Garrido-Ardila EM. Ear therapy and massage therapy in the elderly with dementia: a pilot study. J Tradit Chin Med. 2013; 33:461-467. [PubMed: 24187866]

77. Nelson NL. Massage therapy understanding the mechanisms of action on blood pressure. A scoping review. J Am Soc Hypertens. 2015; 9:785-793. [PubMed: 26324746]

78. Guan L, Collet JP, Yuskiv N, Skippen P, Brant R, Kissoon N. The effect of massage therapy on autonomic activity in critically ill children. Evid Based Complement Altern Med. 2014 Epub ahead of print.

79. Fazeli MS, Pourrahmat MM, Liu M, Guan L, Collet JP. The effect of head massage on the regulation of the cardiac autonomic nervous system: a pilot randomized crossover trial. J Altern Complement Med. 2016; 22:75-80. [PubMed: 26562003]

80. Adib-Hajbaghery M, Rajabi-Beheshtabad R, Abasi A. Effect of whole body massage by patient's companion on the level of blood Cortisol in coronary patients. Nurs Midwifery Stud. 2013; 2:10 15. [PubMed: 25414870] 


\section{Table 1}

Prenatal and postnatal development conditions that are improved by massage therapy: reference numbers, trial types, comparison groups and primary results.

\begin{tabular}{|c|c|c|c|c|}
\hline Conditions & Reference & Trial type & Comparison group & Primary results \\
\hline \multicolumn{5}{|l|}{ Prenatal Development } \\
\hline In vitro fertilization & 2 & RCT & control & > pregnancy rates \\
\hline Prenatal depression & 3 & RCT & yoga & $<$ depression \& prematurity \\
\hline \multicolumn{5}{|l|}{ Infants } \\
\hline \multirow[t]{9}{*}{ Preterm infants } & 4 & RCT & usual care & > weight and height \\
\hline & 5 & RCT & non-oil & >weight \\
\hline & 6 & RCT & usual care & >vagal activity \\
\hline & 7 & $\mathrm{RCT}$ & usual care & >\# natural killer cells \\
\hline & 8 & META & & $5-6$ days $<$ intensive care \\
\hline & 9 & RCT & usual care & $>$ cognitive scores $(12 \mathrm{mos})$ \\
\hline & 11 & RCT & exercise & >vagal activity \\
\hline & 12 & RCT & coconut oil & >oxygen saturation \\
\hline & 13 & $\mathrm{RCT}$ & control & NICU nurses $<$ stressed \\
\hline \multirow[t]{6}{*}{ Full-term infants } & 14 & $\mathrm{RCT}$ & usual care & >attachment \\
\hline & 15 & RCT & no lotion & >sleep patterns \\
\hline & 16 & $\mathrm{RCT}$ & usual care & $<$ bilirubin levels \\
\hline & 17 & $\mathrm{RCT}$ & usual care & $<$ need for phototherapy \\
\hline & 18 & RCT & usual care & <cortisol levels \\
\hline & 19 & $\mathrm{RCT}$ & usual care & $>$ feeding interactions \\
\hline \multicolumn{5}{|l|}{ Children } \\
\hline \multirow[t]{2}{*}{ Autism spectrum disorder } & 21 & REVIEW & & >sleep patterns \\
\hline & 22 & Single arm & & >oxytocin levels \\
\hline
\end{tabular}




\section{Table 2}

Skin conditions that are improved by massage therapy: reference numbers, trial types, comparison groups and primary results.

\begin{tabular}{lllll}
\hline Skin conditions & Reference & Trial & Comparison & Primary results \\
\hline Cleft lip scars & 23 & single arm & & $>$ symmetry \\
Burn scar & 24 & RCT & usual care & <scar tissue \\
Surgical scar & 25 & REVIEW & & >appearance \\
\hline
\end{tabular}




\section{Table 3}

Pain syndromes that are improved by massage therapy: reference numbers, trial types, comparison groups and primary results.

\begin{tabular}{|c|c|c|c|c|}
\hline Pain syndromes & Reference & Trial & Comparison & Primary results \\
\hline \multirow[t]{2}{*}{ Muscle pain } & 27 & $\mathrm{RCT}$ & exercise & >upper extremity function \\
\hline & 28 & $\mathrm{RCT}$ & exercise & $>$ range of motion \\
\hline Labor pain & 29 & $\mathrm{RCT}$ & vibration & $<$ pain and shorter labor \\
\hline Foot & 30 & $\mathrm{RCT}$ & ultrasound & $<$ pain \\
\hline \multirow[t]{3}{*}{ Knee arthritis pain } & 31 & $\mathrm{RCT}$ & exercise & $<$ pain and >performance \\
\hline & 32 & Single arm & & $<$ pain and > gait speed \\
\hline & 33 & $\mathrm{RCT}$ & waitlist & $>$ range of motion \\
\hline Pelvic pain & 34 & $\mathrm{RCT}$ & exercise & $<$ pain intensity \\
\hline Carpal tunnel & 35 & Single arm & & $<$ pain and greater function \\
\hline Hand pain & 36 & $\mathrm{RCT}$ & no analgesic & $<$ pain and > grip strength \\
\hline Arthritis upper limbs & 37 & $\mathrm{RCT}$ & light pressure & $>$ range of motion \\
\hline \multirow[t]{3}{*}{ Neck pain } & 38 & $\mathrm{RCT}$ & light pressure & $>$ range of motion \\
\hline & 39 & META & & $<$ pain \\
\hline & 40 & META & & $<$ pain \\
\hline \multirow[t]{3}{*}{ Back pain } & 41 & RCT & physical therapy & $<$ pain and $<$ disability \\
\hline & 42 & $\mathrm{RCT}$ & Thai massage & $<$ pain and $<$ disability \\
\hline & 43 & $\mathrm{RCT}$ & usual care & $<$ pain \\
\hline Different joints & 44 & REVIEW & & $<$ pain \\
\hline \multirow[t]{2}{*}{ Fibromyalgia } & 45 & META & & $<$ pain \& < sleep disturbance \\
\hline & 46 & META & & $<$ pain \\
\hline Veterans & 48 & Single arm & & $<$ pain \& < anxiety \\
\hline \multirow[t]{3}{*}{ Coronary bypass } & 49 & $\mathrm{RCT}$ & usual care & $<$ pain \\
\hline & 50 & $\mathrm{RCT}$ & rest & $<$ muscle tension \\
\hline & 51 & RCT & handholding & $<$ muscle tension \\
\hline
\end{tabular}




\section{Table 4}

Blood pressure and hypertension conditions that are improved by massage therapy: reference numbers, trial types, comparison groups and primary results.

\begin{tabular}{lllll}
\hline Condition & Reference & Trial & Comparison & Primary results \\
\hline Coronary care & 52 & RCT & usual care & lower systolic \& diastolic blood pressure \\
& 53 & RCT & usual care & lower systolic blood pressure \\
& 54 & RCT & usual care & lower systolic \& diastolic blood pressure \\
& 55 & RCT & mass by relative & lower Cortisol \\
Prehypertension & 56 & RCT & relaxation & lower systolic \& diastolic blood pressure \\
& 57 & RCT & relaxation & lower systolic \&diastolic blood pressure \\
Hypertension & 58 & META & & lower systolic \& diastolic blood pressure \\
\hline
\end{tabular}




\section{Table 5}

Auto-immune and immune conditions improved by massage therapy: reference numbers, trial types, comparison groups and primary results.

\begin{tabular}{lllll}
\hline Condition & Reference & Trial & Comparison & Primary results \\
\hline Auto-immune & & & & \\
Asthma & 59 & RCT & usual care & >forced expiratory flow \\
Multiple sclerosis & 60 & Single arm & & > function \\
& 61 & Single arm & & no change \\
& 62 & RCT & exercise & > balance \\
Immune & & & & \\
HIV-exposed infants & 63 & RCT & usual care & >mental development at 9 mos \\
HIV adults with depression & 64 & RCT & usual care & <depression \\
Cancer in children & 65 & Single arm & & <pain and less anxiety \\
& 66 & RCT & usual care & < vomiting \\
Cancer in adults & 67 & Single arm & & <nausea and vomiting \\
Breast cancer & 68 & RCT & usual care & <sleep disturbance \\
& 69 & RCT & usual care & >immune function \\
Leukemia & 71 & RCT & usual care & < stress \\
Cancer & 72 & META & usual care & < pain \\
\hline
\end{tabular}


Table 6

Aging conditions improved by massage therapy: references, trials, comparison groups and primary results.

\begin{tabular}{lllll}
\hline Condition & Reference & Trial & Comparison & Primary results \\
\hline Postmenopause & 73 & RCT & no odor & <psychological symptoms \\
& 74 & RCT & waitlist & >bone formation marker \\
Parkinson's & 75 & Single arm & & > gait speed \\
Dementia & 76 & Single arm & & <sleep disturbance \\
\hline
\end{tabular}

